\title{
Soft Gel-free ECG electrodes based on Biocompatible Coconut-Oil and Carbon Black
}

\author{
Leonardo A. García-García*, Paul. M. Kreitmar*, Alexander J. Johnson*, Júlio C. Costa*, Arash Pouryazdan*, \\ Pasindu Lugoda ${ }^{\ddagger}$, Daniel Roggen* and Niko Münzenrieder*† \\ * Sensor Technology Research Centre, University of Sussex. Falmer, Brighton BN1 9QT, U.K. \\ ${ }^{\dagger}$ Faculty of Science and Technology, Free University of Bozen-Bolzano, Bozen 39100, Italy. \\ $\ddagger$ Medical Engineering Design Research Group, Nottingham Trent University, Nottingham NG1 4FQ, U.K.
}

\begin{abstract}
Recent developments in telemedicine have caused significant interest in the prolonged monitoring of bioelectric signals. This drives the search for easy-to-use, biocompatible, and environmentally friendly alternatives to conventional resistive wet electrodes. Here we demonstrate the use of CoconutOil and Carbon Black based stretchable electrodes to monitor electrophysiological signals without the need for conductive gels. The developed material is embedded into an elastomer matrix, exhibits a specific resistance $\rho$ of $33.2 \pm 12.3 \Omega \mathrm{m}$, high conformability, and a stretchability up to $1500 \%$. The realised epidermal electrodes were used to record Electrocardiographic (ECG) signals in a 3-lead configuration and compared to commercial wet electrodes. Even after being elongated by $100 \%$ for $\mathbf{1 0 0}$ stretch/release cycles, a reliable recording of the QRS-complex is demonstrated without the need for any contact enhancing or skin irritating substances, proving its potential use in long term ECG monitoring applications.
\end{abstract}

Index Terms-ECG electrodes, biocompatible electrodes, stretchable electrodes, remote health monitoring, telemedicine

\section{INTRODUCTION}

Advanced materials and fabrication techniques enable the realisation of flexible electronic components [1], which are able to provide a platform to create and operate unobtrusive systems close to the human body [2]. These include wearable sensor arrays [3], smart textiles [4], or artificial skins [5] to monitor e.g. motion [6], shape [7], or health [8]. Many of these systems must be soft and stretchable to adapt to the movement of the skin, as well as biocompatible to enable a long lasting contact [9]. This is particularly relevant for electrophysiological measurements relying on electrodes in intimate contact with the skin [10]. Here, Electroencephalography (EEG) [11] and Electrocardiography (ECG) signals, used for heart diseases diagnoses, health monitoring [12], or human identification [13], [14] are the most prominent examples. Such measurements are traditionally done using adhesive wet silver-silver chloride $(\mathrm{Ag} / \mathrm{AgCl})$ electrodes. However, these are not suited for long term monitoring as the gel dries, causing contact problems and skin irritation [15]. Alternative materials such as networks of flexible resistive ECG with silver nanowires [16], Carbon nanotubes in a PDMS matrix [17], organohydrogels [18], or hydrophilic textiles, clad with reduced Graphene oxide (rGO) [19], have been used. However,

This work was partially funded by EPSRC, GCRF, and NIHR, under the contact number: EP/R013837/1 (SmartSensOtics).

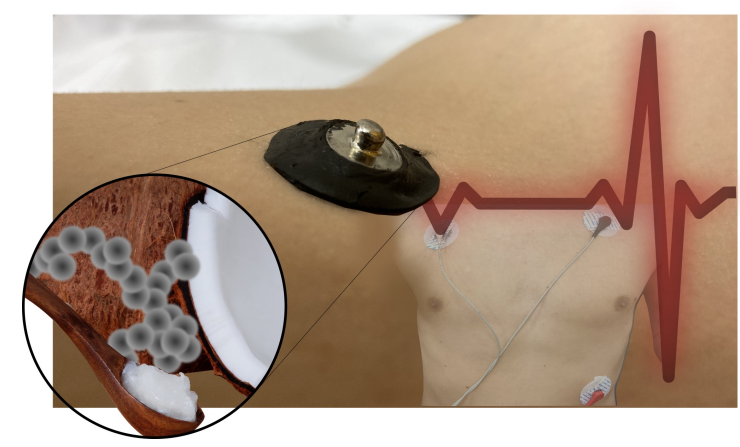

Fig. 1. Ecofriendly and biocompatible ECG electrodes fabricated with a Coconut-oil-Carbon-Black-silicone elastomer.

these reported weak signal acquisition or the requirement of a wet reference electrode. Consequently, alternative easy to use biocompatible electrodes with low contact resistance able to withstand strains induced by deforming skin $(\approx 70 \%)$ [20] are required. In addition, these one-use electrodes should be low-cost and eco-friendly.

Here we present the use of a coconut-oil (CNO) based elastomer [21] with a high concentration of Carbon Black (CB) particles for the fabrication of highly stretchable ECG electrodes (Figure 1). We report the electrical and mechanical characterisation of the material, and compare its suitability for ECG measurements with state of the art $\mathrm{Ag} / \mathrm{AgCl}$ electrodes using an ADS1292R ECG setup (Figure 2).

\section{EXPERIMENTAL PROCEDURE}

\section{A. Electrode Fabrication.}

The fabrication process is illustrated in Figure 3a. The composite was fabricated mixing Ecoflex (00-30 Smooth-on, Pennsylvania, United States), Coconut-Oil (CNO) (Pipkin, London, UK), and Carbon Black (CB) (Vulcan P, Cabot, Boston, Massachusetts, United States) with two different ratios, $10: 5: 1.3$ and $10: 5: 1.7$. First, CNO was mixed with $\mathrm{CB}$ and stirred for $0.5 \mathrm{~h}$ at a constant temperature $\left(37^{\circ} \mathrm{C}\right)$ in a hot bath to prevent lumping. Next, Ecoflex part B was added and stirred for $5 \mathrm{~h}$. Afterwards, Ecoflex part A was incorporated and stirred for a further $0.25 \mathrm{~h}$. The solution was degassed and poured into circular 3D printed moulds with $10 \mathrm{~mm}$ radius and $1 \mathrm{~mm}$ depth, also containing the bottom part of a snap button. Once fully cured, the button's top part 


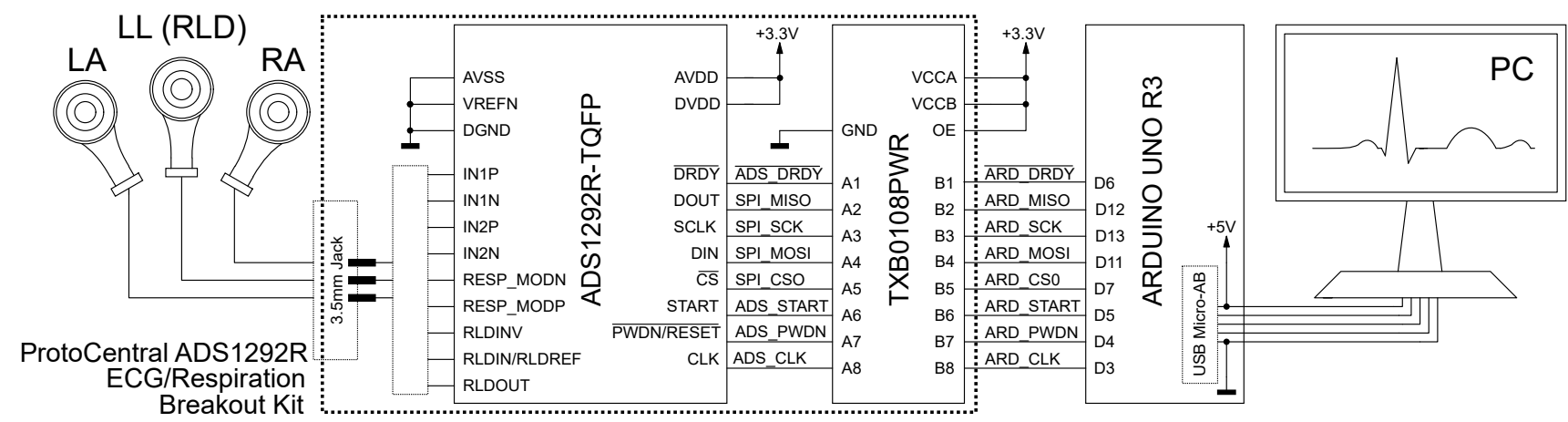

Fig. 2. ECG data recording system. ADS1292R ECG/respiration breakout board connected to an Arduino board. The data sent to a PC over USB COM port.

a

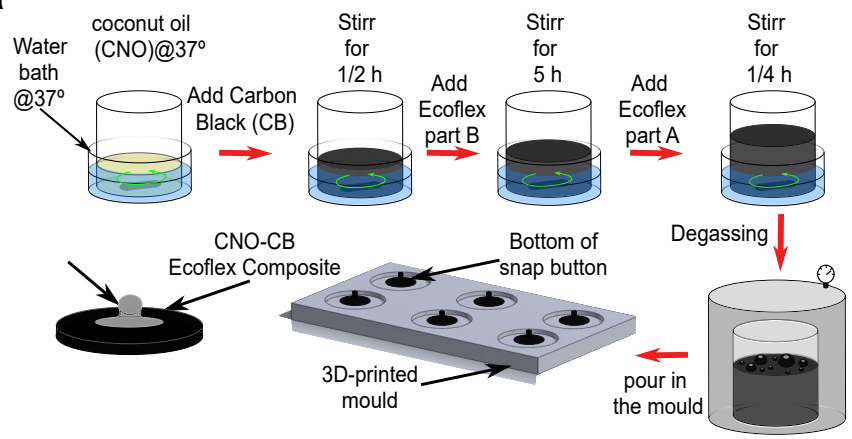

b

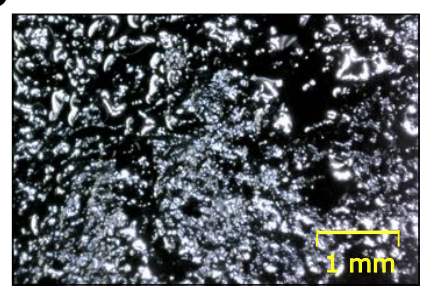

C

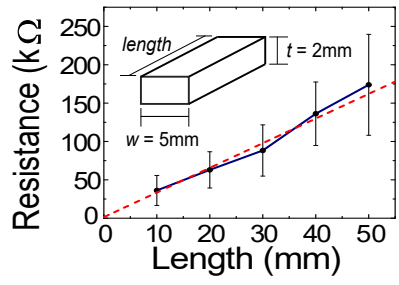

Fig. 3. a) ECG electrode fabrication process. b) Micrograph of the conductive coconut-oil composite. c) Measured average resistance with standard deviation (error bars) and linear fit line (red dotted line).

was snapped, forming a suction cup shape which enhanced the electrodes' attachment to the skin by negative fluid pressure.

\section{B. Data acquisition circuit}

The electrodes were interfaced with a ProtoCentral ADS1292R ECG/ Respiration breakout board, connected to an Arduino board to get the ECG signals as shown in Figure 2. This board includes a differential programmable gain amplifier (PGA), ADC and voltage reference. It was configured to use the internal $2.42 \mathrm{~V}$ reference and a $125 \mathrm{~Hz}$ sampling rate with a gain of 6 . The first 8 bits of the raw ADC data were discarded. Following the manufacturer's recommendations, a 161th order $40 \mathrm{~Hz}$ low pass filter was used in software in combination with the IC's internal oversampling above the set data rate and a digital downsampling to reduce the output rate of the data. A right Leg Drive (RLD) circuit was used to reduce common mode noise. This circuit drives the body with the inverted common mode signal, attempting to actively cancel the common-mode interference. This signal is generated internally by the IC. As this feedback introduces additional noise, a $3 \mathrm{MHz} \mathrm{RC}$ filter was used in the IC.

Einthoven's 3-lead was used on the chest to record the ECG signals [22]. This configuration is commonly used to record the potential between both arms, and between the left leg and each arm, measuring a differential reading with respect to a neutral point, the left leg. In this research this configuration was set up in the chest by placing the electrodes equidistantly to improve the results, as illustrated in the inset in Fig. 4a. The skin was only cleaned with Isopropanol alcohol before placing the electrodes with self adhesive pads and no contact enhancing gel or substances were used.

\section{RESULTS AND DISCUSSION.}

Figure $3 \mathrm{~b}$ shows a micrograph of the material's surface. The material is porous filled with an oily CNO-CB suspension. Consequently, it has a rough oily surface with $\mathrm{CNO}-\mathrm{CB}$ oil droplets smaller than $1 \mathrm{~mm}$ that reduce the contact resistance. Samples with the same width $(5 \mathrm{~mm})$ and thickness $(2 \mathrm{~mm})$ but 5 different lengths (10, 20, 30, 40 and $50 \mathrm{~mm}$ ) were used to determine the specific Resistance. Figure 3c shows a plot of the average contact resistance of the $10.5 \%$ CNO$\mathrm{CB}$ polymer. There is the expected linear relation between length and resistance, but it is also observed that the standard deviation increases for longer samples. This indicates a non homogeneous dispersion of the conductive filler on the polymeric matrix. However, the linear fit shows that the contact resistance is negligible. The resulting intrinsic resistivity is $\rho=33.2 \pm 12.3 \Omega \mathrm{m}$ for $10.5 \%$ wt concentration, while the $7.5 \%$ wt concentration resulted in a significantly higher $\rho$ of $145.8 \pm 71.2 \Omega \mathrm{m}$.

The electrodes are further analysed by taking ECG measurements. Figure 4 shows the resulting ECG recordings taken with the $\mathrm{CNO}-\mathrm{CB}$ electrodes in comparison to the $\mathrm{Ag} / \mathrm{AgCl}$ reference wet gel electrodes. It can be clearly seen that the electrodes with $10.5 \%$ wt CB provide a better signal quality (4b) than the ones with a $7.5 \%$ wt (4a). This is due to the different resistances of the corresponding materials. The $10.5 \% \mathrm{wt}$ $\mathrm{CB}$ electrodes provide a signal strength of $\approx 0.8 \mathrm{mV}$ and a visible QRS-complex similar to those of the wet electrodes, despite not using any conductive gel.

To evaluate the mechanical performance, strain tests were carried out to analyse the maximum strain the electrodes can 
a

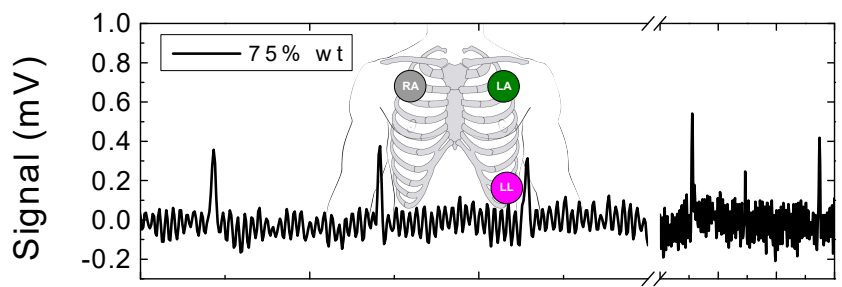

b

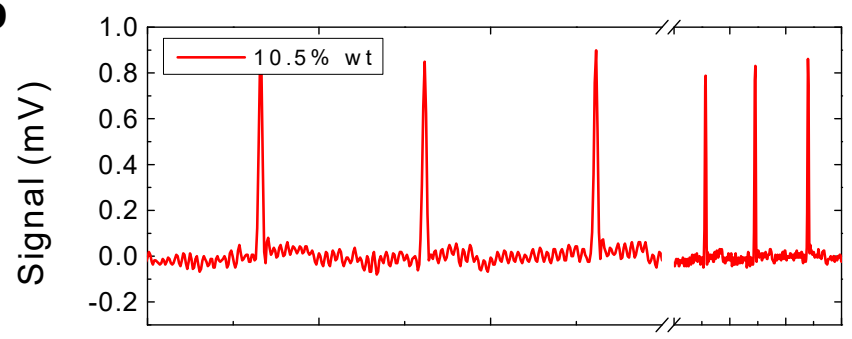

C

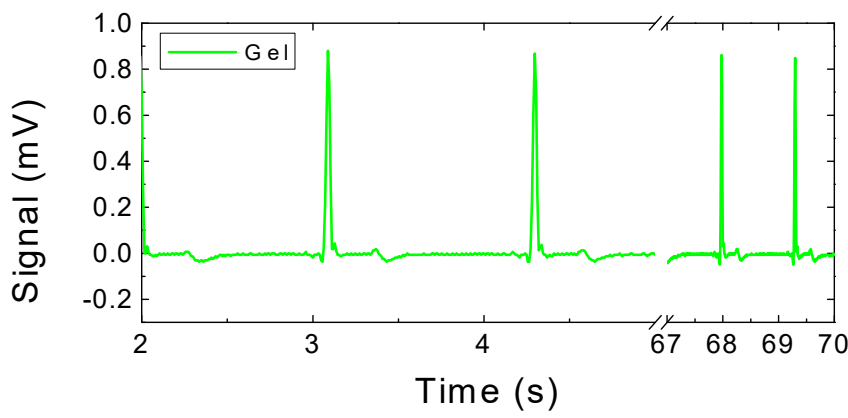

Fig. 4. Comparison of ECG signals taken in a Chest 3-lead configuration with three different materials, a) $7.5 \%$ wt $\mathrm{CB}$ concentration, b) $10.5 \%$ wt $\mathrm{CB}$ concentration, and c) $\mathrm{Ag} / \mathrm{AgCl}$ reference wet gel electrodes.

sustain, as well as their long term resistance to mechanical deformation. 100 stretch/release cycles applying a maximum strain of $100 \%$ (more than the maximum stretchability of

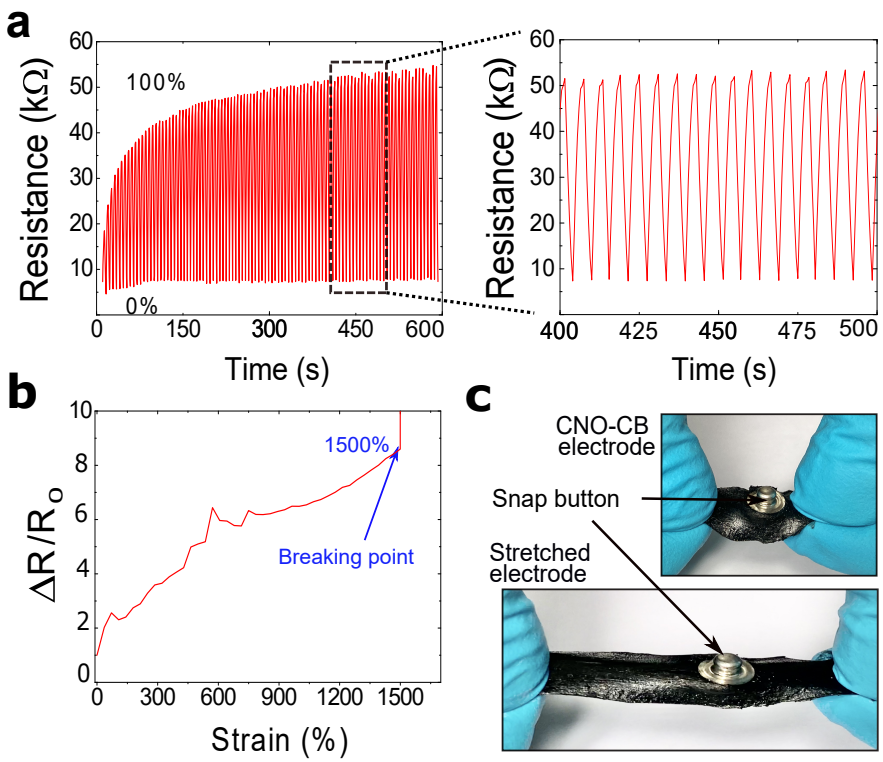

Fig. 5. Characterisation of the $10.5 \%$ wt CB electrode. a) 100 stretch/release cyclic test. b) Strain test to rupture. c) Stretched vs. non stretched electrode. a

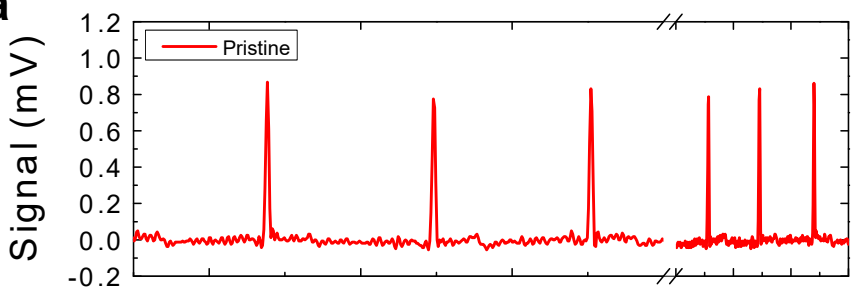

b

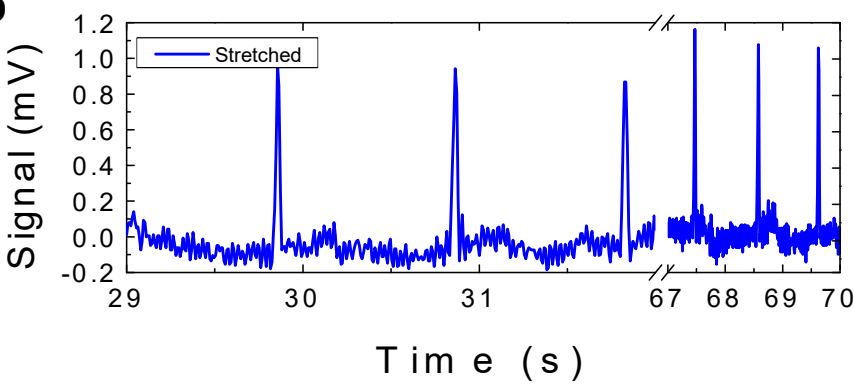

Fig. 6. ECG signals using the CNO-CB electrode. ECG signals acquired with the a) pristine electrode and b) with the electrodes after cyclic test.

skin) were performed on each electrode. Figure 5a shows the cyclic test results of a representative sample, illustrating how the resistance of the relaxed electrode is hardly influenced by cyclic strain. After 100 cycles, the resistance at $0 \%, \mathrm{R}_{0}$, was $5.85 \mathrm{k} \Omega$, representing $0.015 \%$ change, with an average $\mathrm{R}_{0}=7.26 \pm 0.66 \mathrm{k} \Omega$. On the other hand, the resistance at $100 \%, \mathrm{R}_{100}$, was $54.5 \mathrm{k} \Omega$, three times its initial value $(18.4 \mathrm{k} \Omega)$. The maximum stretchability of the material was determined by stretching a material sample until its breaking point while recording the resistance. Figures $5 \mathrm{~b}$ and $5 \mathrm{c}$ show the increase in resistance with strain, yielding an ultimate strain of $1500 \%$. Finally, further ECG measurements were performed to demonstrate the usability of the electrodes after the 100 cycles stretching test. The results are displayed in Figure 6. Although a slightly increase on the signal's amplitude and noise after the cyclic test (Figure 6b) was observed, the QRS-complex still clearly defined, denoting a slightly effect of stretching on the soft coconut-oil electrodes performance.

\section{CONCLUSIONS.}

Biocompatible coconut-oil was used to create flexible and stretchable ECG electrodes. The resulting composite can withstand large strain values and repetitive stretching, remaining functional even after being elongated by $100 \%$ for 100 cycles. The results show that the electrodes are suitable to measure ECG signals without the need for any conductive gels. However, the impedance and artifacts remain slightly higher when compared to conventional $\mathrm{Ag} / \mathrm{AgCl}$ electrodes. Future work will look to improve signal integrity, and the analysis of the electrodes' performance and stability in long-term applications. Nevertheless, the main advantages of the CNOCB electrodes are that they are ecofriendly, the inexpensive fabrication technique, and their potential application for longterm monitoring, as they work reliably without any additional electrolyte gel, thereby preventing problems such as drying and skin irritation. 


\section{REFERENCES}

[1] J. C. Costa, F. Spina, P. Lugoda, L. Garcia-Garcia, D. Roggen, and N. Münzenrieder, "Flexible sensors-from materials to applications," Technologies, vol. 7, no. 2, p. 35, Apr. 2019. [Online]. Available: https://doi.org/10.3390/technologies7020035

[2] A. Nathan, A. Ahnood, M. T. Cole, S. Lee, Y. Suzuki, P. Hiralal, F. Bonaccorso, T. Hasan, L. Garcia-Gancedo, A. Dyadyusha et al., "Flexible electronics: the next ubiquitous platform," Proceedings of the IEEE, vol. 100, no. Special Centennial Issue, pp. 1486-1517, 2012. [Online]. Available: https://doi.org/10.1109/JPROC.2012.2190168

[3] W. Gao, S. Emaminejad, H. Y. Y. Nyein, S. Challa, K. Chen, A. Peck, H. M. Fahad, H. Ota, H. Shiraki, D. Kiriya, D.-H. Lien, G. A. Brooks, R. W. Davis, and A. Javey, "Fully integrated wearable sensor arrays for multiplexed in situ perspiration analysis," Nature, vol. 529, no. 7587, pp. 509-514, Jan. 2016. [Online]. Available: https://doi.org/10.1038/nature16521

[4] P. Lugoda, J. C. Costa, C. Oliveira, L. A. Garcia-Garcia, S. D. Wickramasinghe, A. Pouryazdan, D. Roggen, T. Dias, and N. Münzenrieder, "Flexible temperature sensor integration into e-textiles using different industrial yarn fabrication processes," Sensors, vol. 20, no. 1, p. 73, Dec. 2019. [Online]. Available: https://doi.org/10.3390/s20010073

[5] J. C. Yang, J. Mun, S. Y. Kwon, S. Park, Z. Bao, and S. Park, "Electronic skin: Recent progress and future prospects for skinattachable devices for health monitoring, robotics, and prosthetics," Advanced Materials, vol. 31, no. 48, p. 1904765, Sep. 2019. [Online]. Available: https://doi.org/10.1002/adma.201904765

[6] Q. Gao, J. Zhang, Z. Xie, O. Omisore, J. Zhang, L. Wang, and H. Li, "Highly stretchable sensors for wearable biomedical applications," Journal of Materials Science, vol. 54, no. 7, pp. 5187-5223, Nov. 2018. [Online]. Available: https://doi.org/10.1007/s10853-018-3171-x

[7] P. Lugoda, L. A. Garcia-Garcia, S. Richoz, N. Münzenrieder, and D. Roggen, "ShapeSense3d" in Proceedings of the 2019 ACM International Joint Conference on Pervasive and Ubiquitous Computing and Proceedings of the 2019 ACM International Symposium on Wearable Computers - UbiComp/ISWC '19. ACM Press, 2019. [Online]. Available: https://doi.org/10.1145/3341162.3343846

[8] A. T. Güntner, S. Abegg, K. Königstein, P. A. Gerber, A. SchmidtTrucksäss, and S. E. Pratsinis, "Breath sensors for health monitoring," ACS Sensors, vol. 4, no. 2, pp. 268-280, Jan. 2019. [Online]. Available: https://doi.org/10.1021/acssensors.8b00937

[9] C. Wang, T. Yokota, and T. Someya, "Natural biopolymer-based biocompatible conductors for stretchable bioelectronics," Chemical Reviews, vol. 121, no. 4, pp. 2109-2146, 2021. [Online]. Available: https://doi.org/10.1021/acs.chemrev.0c00897

[10] D. G. Barone and G. G. Malliaras, "Epidermal electrophysiology at scale," Nature Biomedical Engineering, vol. 3, no. 3, pp. 165-166, Mar. 2019. [Online]. Available: https://doi.org/10.1038/s41551-019-0365-8

[11] M. Fatoorechi, J. Parkinson, R. Prance, H. Prance, A. Seth, and D. Schwartzman, "A comparative study of electrical potential sensors and $\mathrm{ag} / \mathrm{AgCl}$ electrodes for characterising spontaneous and event related electroencephalagram signals," Journal of Neuroscience Methods, vol. 251, pp. 7-16, Aug. 2015. [Online]. Available: https://doi.org/10.1016/j.jneumeth.2015.04.013

[12] M. Blanco-Velasco, B. Weng, and K. E. Barner, "ECG signal denoising and baseline wander correction based on the empirical mode decomposition," Computers in Biology and Medicine, vol. 38, no. 1, pp. 1-13, Jan. 2008. [Online]. Available: https://doi.org/10.1016/j.compbiomed.2007.06.003

[13] I. Jekova and G. Bortolan, "Personal verification/identification via analysis of the peripheral ECG leads: Influence of the personal health status on the accuracy," BioMed Research International, vol. 2015, pp. 1-13, 2015. [Online]. Available: https://doi.org/10.1155/2015/135676

[14] L. Biel, O. Pettersson, L. Philipson, and P. Wide, "ECG analysis: a new approach in human identification," in IMTC/99. Proceedings of the 16th IEEE Instrumentation and Measurement Technology Conference (Cat. No.99CH36309), vol. 50, no. 3. IEEE, 2001, pp. 808-812. [Online]. Available: https://doi.org/10.1109/imtc.1999.776813

[15] Y. M. Chi and G. Cauwenberghs, "Wireless non-contact EEG/ECG electrodes for body sensor networks," in 2010 International Conference on Body Sensor Networks. IEEE, Jun. 2010. [Online]. Available: https://doi.org/10.1109/bsn.2010.52
[16] Z. Cui, Y. Han, Q. Huang, J. Dong, and Y. Zhu, "Electrohydrodynamic printing of silver nanowires for flexible and stretchable electronics," Nanoscale, vol. 10, pp. 6806-6811, 2018. [Online]. Available: http://dx.doi.org/10.1039/C7NR09570H

[17] J. H. Kim, J.-Y. Hwang, H. R. Hwang, H. S. Kim, J. H. Lee, J.-W. Seo, U. S. Shin, and S.-H. Lee, "Simple and cost-effective method of highly conductive and elastic carbon nanotube/polydimethylsiloxane composite for wearable electronics," Scientific Reports, vol. 8, no. 1, Jan. 2018. [Online]. Available: https://doi.org/10.1038/s41598-017-18209-w

[18] Q. Wang, X. Pan, C. Lin, D. Lin, Y. Ni, L. Chen, L. Huang, S. Cao, and X. Ma, "Biocompatible, self-wrinkled, antifreezing and stretchable hydrogel-based wearable sensor with pedot:sulfonated lignin as conductive materials," Chemical Engineering Journal, vol. 370, pp. 1039-1047, 2019. [Online]. Available: https://doi.org/10.1016/j.cej.2019.03.287

[19] M. K. Yapici, T. Alkhidir, Y. A. Samad, and K. Liao, "Graphene-clad textile electrodes for electrocardiogram monitoring," Sensors and Actuators B: Chemical, vol. 221, pp. 1469-1474, 2015. [Online]. Available: https://doi.org/10.1016/j.snb.2015.07.111

[20] N. Münzenrieder, D. Karnaushenko, L. Petti, G. Cantarella, C. Vogt, L. Büthe, D. D. Karnaushenko, O. G. Schmidt, D. Makarov, and G. Tröster, "Entirely flexible on-site conditioned magnetic sensorics," Advanced Electronic Materials, vol. 2, no. 8, p. 1600188, Jun. 2016. [Online]. Available: https://doi.org/10.1002/aelm.201600188

[21] P. Lugoda, J. C. Costa, L. A. Garcia-Garcia, A. Pouryazdan, Z. Jocys, F. Spina, J. Salvage, D. Roggen, and N. Münzenrieder, "Coco stretch: Strain sensors based on natural coconut oil and carbon black filled elastomers," Advanced Materials Technologies, vol. 6, no. 2, p. 2000780, Dec. 2020. [Online]. Available: https://doi.org/10.1002/admt.202000780

[22] G. Gargiulo, P. Bifulco, M. Cesarelli, A. McEwan, H. Moeinzadeh, A. O'Loughlin, I. Shugman, J. Tapson, and A. Thiagalingam, "On the einthoven triangle: A critical analysis of the single rotating dipole hypothesis," Sensors, vol. 18, no. 7, p. 2353, Jul. 2018. [Online]. Available: https://doi.org/10.3390/s18072353 